\title{
EDITORIAL
}

\section{The MRC Needs for Care Assessment: progress and controversies ${ }^{1}$}

In the 1980s researchers at the Medical Research Council Social Psychiatry Unit developed and refined methods for measuring needs for psychiatric care. Building upon the initial studies of Wykes et al. (1982) and Wykes \& Sturt (1986), the most recent and highly researched instrument is the MRC Needs for Care Assessment (Brewin et al. 1987; Brewin \& Wing, 1989). In this commentary we will review some of the knowledge that has been gained about this instrument and discuss some recent controversies concerning its use.

The MRC Needs for Care Assessment was developed specifically for measuring the needs of the long-term mentally ill living in the community and in touch with psychiatric services. It involves two distinct stages of data collection. In the first information is sought from the best-placed sources, who may include members of staff, relatives, and the patients themselves, about the presence of problems in 20 areas of clinical and social functioning. In the second stage a member of staff is questioned about a standard list of items of care relevant to each identified problem. Questioning includes details of whether each item of care has been tried, and about its appropriateness, effectiveness, and acceptability to the patient. Judgements of the primary need status (met need, unmet need, no need, or no meetable need) follow algorithmically from these ratings. In order that the assessment should not be artificially constrained by current practice or resources, resulting in over-conservative judgements, raters are instructed not to take account of any training or resource implications. The assessment is thus explicitly concerned with normative needs that are independent of those of any particular service setting. Both in conception and use the instrument aims to set minimum standards of care based on a workable synthesis of current knowledge and practice rather than to strive for some notional ideal care.

From the outset we were clear that judgements indicating a mismatch between the care recommended by the assessment and the care actually provided ('unmet need ' and 'overprovision') can only be 'recommendations for action' (Brewin et al. 1987, p. 980). In other words, it is not possible at one assessment to specify exactly the nature of a patient's needs, but only to make recommendations consistent with good practice. Whether there is actually an unmet need or an overprovision will depend on staff actually offering or withdrawing the relevant item of care. Only by this means is it possible to establish that a recommended action is effective and acceptable to the patient, or that an item of care can be discontinued without harm. A comprehensive needs assessment, then, usually depends on completing the audit cycle, i.e. assessing outcomes in order to evaluate the success of any interventions based on the initial assessment.

The quality of the initial assessment depends in part on taking account of the acceptability of specific items of care, and in the manual interviewers are instructed to enquire about this. For some groups of patients, such as those who are homeless or out of contact with psychiatric services, this will of course be particularly important. Extension of the MRC Needs for Care Assessment methodology to these groups is therefore likely to require additional questioning to establish acceptability and to take account of patients' responses to past interventions.

Another important issue relates to the guiding philosophy of care incorporated in the assessment. All needs assessments incorporate, whether explicitly or implicitly, a philosophy of care, and the results of the assessment have to be interpreted in that light. This means that an assessment should not be selected unless the users are in broad agreement with the model of care it incorporates. The

1 Address for correspondence. Professor J. K. Wing, The Royal College of Psychiatrists, Research Unit, 17 Belgrave Square, London SWIX 8PG. 
principles of the MRC Needs for Care Assessment (described in Brewin et al. 1987) are designed to equip people as far as possible for independent life in the community. Any symptom, behaviour problem, or lack of competence at a skill of daily living likely to disadvantage them is therefore a potential target for intervention. Following this principle, the assessment targets skill deficits such as inability to cook and shop even though the patient's immediate requirements are being met by relatives or care staff. The rationale is that in the long run it is preferable to maximize the patient's skills in order to have them functioning at the highest level possible and able to cope with disruptions to their normal care arrangements. This is likely to have the additional benefit of reducing the burden on relatives, other carers, or fellow residents and removing potential sources of criticism and distress in their relationships. In any sample, however, there are likely to be some patients who are so impaired that they are unlikely to be able to live independently, and the manual describes how to rate them.

In the most recent version (Brewin \& Wing, 1989) there are a number of minor changes to the version described in Brewin et al. $(1987,1988)$. The original item on decision-making in Section B is omitted, reducing the total number of areas of functioning to 20. Item B12 (Occupational skills) is now only completed if sheltered or open employment is considered appropriate and possible. In other words, the section is not applicable if the person is too old for employment, is a housewife who is not seeking employment, or if the nature of any handicap is so severe as to rule out employment.

In the original version it was not possible to record a met need and a future need for the same item. The results reported in Brewin et al. (1988) accord met needs the priority, so that future needs were only recorded when no items of care were currently being offered. In the most recent version both a met need and a future need may be recorded for the same item, as they are rated independently.

A final change is the introduction of a fourth primary need status - 'no meetable need'. This describes situations where there is disablement but no action is appropriate and feasible. In previous versions these situations were included with cases in which there was no disablement under the general heading of 'no need', but this was generally felt to be unsatisfactory. The new category clearly identifies areas where patients have problems but no action is currently feasible.

\section{EVIDENCE FOR RELIABILITY AND VALIDITY}

Initial data on the reliability and validity of the assessment were presented in Brewin et al. (1987, 1988). Clearly, estimates of validity can only be limited ones given that by definition need involves the making of value judgements and that no other comparable definitions and measures of need exist. Validity is also likely to be limited by cultural factors, such as the appropriateness of acquiring such skills as cooking and housework (see Lesage et al. 1991). Perhaps the most practical approach to validity would be to have a group of patients independently assessed before and after an intervention phase in which the Needs for Care assessment was used to structure the care provided. Ideally, patients would be randomly assigned to structured needs assessment versus a traditional review of their treatment.

Some recent data do, however, shed light on the reliability of the procedure. Lesage et al. (1991) employed an Italian version of the assessment in a study of psychiatric services in South Verona. The primary need status of 61 identified problems found in 20 patients was independently rated by one Camberwell-trained judge and by two Italian judges. The three judges showed a high level of agreement (kappa $=0.92, P<0.0001$ ), correctly identifying a relatively high level of unmet need among this subset of problems.

The needs of patients from two different geographical areas attending the same day hospital have recently been assessed in two independent studies. One group $(N=11)$ was assessed as part of the Camberwell High Contact Study (Brewin et al. 1988) and the other $(N=66)$ as part of a survey of day care in an inner city (Wainwright et al. 1988). Both studies used the MRC Needs for Care Assessment, although whereas Brewin et al. had a team of independent investigators interviewing 
patient, day staff, and relatives, Wainwright et al. adapted the assessment for use by a single investigator interviewing patient and day staff only. Despite these procedural differences the results of the two studies were highly similar. Brewin et al. identified a mean of $5 \cdot 27$ clinical and social problems per patient, of which $28 \%$ were rated as unmet needs. Wainwright et al. identified on average 6.4 problems per patient, of which $30 \%$ were rated as unmet needs.

Van Haaster et al. (1994) examined the reliability and validity of a French translation of the instrument in a study of 98 short-term and long-term patients in Montreal. Only items where the primary need status was met or unmet were considered, in order that agreement should not be artificially inflated by the large number of items where there was no need. The reliability study was based on two independent judges making 327 ratings on the data from 33 patients. Kappa was 0.88 and the agreement rate $96 \%$, with little variation due to length of stay or type of problem. Judgements on the needs of 80 of the patients in the sample were in agreement with the views of the treating psychiatrist on $93 \%$ of occasions. Almost half the disagreements were due to a change in the clinical state of the patient between the time of the research assessment and the time psychiatrists completed their questionnaires. Agreement between psychiatrists and the research team on unmet needs, i.e. judgements that indicated discrepancies between ideal and actual care, was slightly lower at $83 \%$.

In summary, these three studies suggest that the measure has good reliability in the hands of suitably trained investigators, and is robust enough to tolerate minor procedural deviations.

\section{TRAINING AND MANPOWER REQUIREMENTS}

The judgements required of assessors involve familiarity both with long-term patients and their characteristic problems, and with the specific items of care that are considered. Thus, it is not suitable for investigators who have not had clinical experience of this patient group, unless considerable extra training is provided. The conclusion of those who have used it is that it is best completed by a clinical team or by a research team that includes members with varying professional backgrounds. Psychiatric and psychological expertise are of prime importance. All users of the instrument should first attend an authorized training programme to familiarize themselves with the instrument. At present this consists of two half-day workshops, with an intervening period in which users gain first-hand experience of carrying out assessments.

Data collection need not be lengthy if the instrument is used to shape the initial assessments conducted by a clinical team. Almost all the information is of the kind that should be routinely collected on admission, and this should be adequate for most clinical purposes. However, if comparability with other units or clinical teams is necessary for research purposes, additional staff would become necessary. This may well involve the addition of standardized assessments of functioning such as the Social Behaviour Schedule (Wykes \& Sturt, 1986). Data collection will take considerably longer when the instrument is used by research investigators, as in the Camberwell High Contact Survey. Interviewing patients, day staff, relatives, and other involved persons where appropriate, summarizing the medical records, and combining inconsistent information, require substantial resources.

\section{CONTROVERSIES IN THE ASSESSMENT OF NEED}

A number of controversies have recently arisen, principally concerned with the measurement of need in other groups of psychiatric patients. Pryce et al. (1993) used the original version of the Needs for Care Assessment with psychiatric in-patients and had difficulty in rating need for social and personal skills in some patients who were old and/or severely handicapped. In order to make the instrument more suitable for this patient group they proposed rating certain areas as "not applicable', to mean 'skill or ability seems unlikely ever to be required because of age and/or severity of handicap'. In their study over a third of ratings of need for social and personal skills were 
judged to be 'not applicable'. Around $60 \%$ of these ratings were confined to 47 of the oldest and most disabled patients, who each received from 7 to 9 'not applicable' ratings.

In our view 'not applicable' ratings should be reserved for areas such as employment skills or household management skills that cannot be regarded as relevant to very old or severely disabled patients, and where no provision of any kind is required. In the other areas such as shopping or cooking provision still has to be made, even though it may be unrealistic to expect severely disabled patients to exercise these skills themselves. Where disability rules out the exercise of these skills the appropriate rating is for a 'met need' (for a staffed hostel or other sheltered residence, for example).

Pryce $e t$ al. also noted that other, less disabled patients in their sample who lived on less wellstaffed wards received a high number of ratings of unmet need for assessment, reflecting the lack of opportunity to exercise these skills and staff's lack of knowledge about their capabilities. They raised the general question of whether skills training is worthwhile for psychiatric patients who are fairly severely handicapped and need sheltered accommodation. This is an area of some controversy, but we believe it is important to reach an independent consensus on what constitutes a good standard of care rather than let this be determined by the resources available locally.

Our opinion, based on the considerations given above and on what is now known about the effects of institutionalization, is that it is desirable for patients to be free to exercise as many skills as they are capable of, whether they are living as in-patients or in the community. The issue of whether resources are available to achieve this should be kept distinct. If patients are able to use a skill but are prevented from doing so there is therefore overprovision of care. If their level of skill is unknown, there is a need for assessment. Thus, the numerous ratings of 'level of functioning not known' and 'unmet need for assessment' recorded by Pryce et al. are, we believe, valuable in drawing attention to areas in which a higher standard of care might be attainable.

Another recent article commented about the use of the MRC Needs for Care Assessment with the homeless mentally ill (Hogg \& Marshall, 1992). Although Hogg and Marshall acknowledged that the assessment did broadly reflect conditions in the hostels they studied, they criticized the quality of the information provided by the assessment and suggested that the method failed to take sufficient account of the views of staff and relatives. They first noted that identified needs for structured behaviour programmes for socially embarrassing behaviour have resource implications, which are explicitly ignored by the assessment for reasons discussed above. They then suggested that:

The decision of whether or not to proceed with such programmes depends largely on the effects the socially embarrassing behaviour is having on hostel staff and the residents themselves. In some cases a behaviour which in most community settings would be unacceptable, has a minimum effect in this particular setting - it may even be adaptive, for example when a homeless woman shouts and swears at strange men (p. 1033).

Hogg \& Marshall also note that the hostels are characterized by tolerance and that care must be taken in altering their atmosphere.

Passing over the example of the woman who shouts and swears, which seems to stretch the meaning of the word 'adaptive' since this behaviour might invite a reciprocal aggression, we understand the authors' dilemma but do not agree with their solution. The authors say that several residents have problems that might be helped by methods that have not been tried but that it would spoil the milieu to introduce them. They wish, therefore, to change the rating system in order to allow them to say that there is no need.

There are certainly occasions when such people need a non-demanding environment. But our system also allows the rating of a future need, with a concomitant requirement to continue assessment to discover the right moment to suggest more active help, perhaps in a different milieu. If, when that time comes, the appropriate milieu is not available, there is an unmet need. The system is intended to highlight such gaps in provision. We do not pretend that it can solve problems of priority or cost, but the ratings can help to inform those who have to make such decisions.

The authors' solution, however, is to introduce a different philosophy of care. Their aim is to achieve behaviour acceptable in the hostels they studied, rather than in the community more 
broadly. Unmet needs for help that might foster more independence are thus seen as irrelevant; not because our rating system is at fault, but because our goal is rejected. They may not have understood that their argument can equally be applied to any situation in which a temporary solution to a problem has been found but where, given adequate resources, a better long-term solution is possible though unavailable. Thus, they restrict the concept of need to what is provided, rather than to what would be most appropriate to the circumstances.

The third critical point made by Hogg \& Marshall is that the needs assessment schedule:

makes no attempt to elicit the client's opinion as to whether or not they are interested in receiving certain items of care. When this is combined with the fact that due to 'environmental constraints' we often do not know if a client has a problem in an area of functioning (with getting meals for example), a major assessment problem is created. The danger is that many will be rated as having needs for assessment or for items of care they do not want (p. 1033).

This comment ignores the fact that users of the assessment are explicitly enjoined to take account of clients' views about the acceptability of items of care. Moreover, Hogg \& Marshall themselves do not appear to have asked their clients about their skills and abilities, even though they are usually reliable sources of information (MacCarthy et al. 1986). Instead, they relied on the knowledge of staff, despite the lack of opportunities for appropriate observations at the hostel. Thus it seems to us that more comprehensive questioning by the investigators of the clients would have substantially increased the quality of the needs assessments and simultaneously answered many of the criticisms wrongly directed at the schedule.

To summarize, two general issues that arise from Pryce et al.'s (1993) and Hogg \& Marshall's (1992) articles are relevant to many of the needs assessment studies with which we are familiar. First, investigators must be clear before they commence their study about their preferred philosophy of care, and must choose a compatible instrument. Secondly, assessments are only as good as the quality of the information collected. We remain confident that, properly used, the MRC Needs for Care Assessment is a reliable tool and has a valuable part to play in assessing the needs of a wide variety of people with mental disorder.

C. R. BREWIN AND J.K. WING

\section{REFERENCES}

Brewin, C. R. \& Wing, J. K. (1989). Manual of the MRC Needs for Care Assessment. Memo, MRC Social Psychiatry Unit, London. (Available from Professor C. R. Brewin.)

Brewin, C. R., Wing, J. K., Mangen, S. P., Brugha, T. S. \& MacCarthy, B. (1987). Principles and practice of measuring needs in the long-term mentally ill ; the MRC Needs for Care Assessment. Psychological Medicine 17, 971-982.

Brewin, C. R., Wing, J. K., Mangen, S., Brugha, T. S., MacCarthy, B. \& Lesage, A. D. (1988). Needs for care among the long-term mentally ill. A report from the Camberwell High Contact Survey. Psychological Medicine 18, 457-468.

Hogg, L. I. \& Marshall, M. (1992). Can we measure need in the homeless mentally ill? Using the MRC Needs for Care Assessment in hostels for the homeless. Psychological Medicine 22, 1027-1034.

Lesage, A. D., Mignolli, G., Faccincani, C. \& Tansella, M. (1991). Standardised assessment of the needs for care in a cohort of patients with schizophrenic psychoses. In Community-based Psychiatry: Long-term Patterns of Care in South-Verona (ed. M Tansella), pp. 27-33. Psychological Medicine Monograph Supplement 19. Cambridge University Press: Cambridge.

MacCarthy, B., Benson, J. \& Brewin, C. R. (1986). Task motivation and problem appraisal in long-term psychiatric patients. Psychological Medicine 16, 431-438.
Pryce, I. G., Griffiths, R. D., Gentry, R. M., Hughes, I. C. T., Montague, L. R., Watkins, S. E., Champney-Smith, J. \& McLackland, B. M. (1993). How important is the assessment of social skills in current long-stay in-patients? British Journal of Psychiatry 162 , 498-502.

van Haaster, I., Lesage, A. D., Cyr, M. \& Toupin, J. (1994). Further reliability and validity studies with the Needs for Care Assessment Schedule. Psychological Medicine 24 (in the press).

Wainwright, T., Holloway, F. \& Brugha, T. S. (1988). Day care in an inner city. In Community Care in Practice: Services for the Continuing Care Client (ed. A. Lavender and F. Holloway), pp. 231-256. Wiley: Chichester.

Wykes, T. \& Sturt, E. (1986). The measurement of social behaviour in psychiatric patients: an assessment of the reliability and validity of the SBS schedule. British Journal of Psychiatry 148, 1-11.

Wykes, T., Creer, C. \& Sturt, E. (1982). Needs and the deployment of services. In Long-term Community Care. Experience in a London Borough (ed. J. K. Wing). Psychological Medicine Monograph Supplement, 2. Cambridge University Press: Cambridge. 